\title{
Modifying Adolescent Interpretation Biases Through Cognitive Training: Effects on Negative Affect and Stress Appraisals
}

\author{
Machteld D. Telman • Emily A. Holmes • \\ Jennifer Y. F. Lau
}

Published online: 31 May 2013

(c) The Author(s) 2013. This article is published with open access at Springerlink.com

\begin{abstract}
Adolescent anxiety is common, impairing and costly. Given the scale of adolescent anxiety and its impact, fresh innovations for therapy are in demand. Cognitive Bias Modification of Interpretations (CBM-I) studies of adults show that by training individuals to endorse benign interpretations of ambiguous situations can improve anxious moodstates particularly in response towards stress. While, these investigations have been partially extended to adolescents with success, inconsistent training effects on anxious moodstates have been found. The present study investigated whether positive versus negative CBM-I training influenced appraisals of stress, in forty-nine adolescents, aged 15-18. Data supported the plasticity of interpretational styles, with positively-trained adolescents selecting more benign resolutions of new ambiguous situations, than negativelytrained adolescents. Positively-trained adolescents also rated recent stressors as having less impact on their lives than negatively-trained adolescents. Thus, while negative styles may increase negative responses towards stress, positive styles may boost resilience.
\end{abstract}

M. D. Telman · J. Y. F. Lau

Department of Experimental Psychology, University of Oxford, Oxford OX1 4AU, UK

J. Y. F. Lau

e-mail: jennifer.lau@psy.ox.ac.uk

M. D. Telman ( $\square)$

Department of Clinical Child and Family Studies, Faculty of Psychology and Education, VU University Amsterdam, Van der Boechorststraat 1, 1081 BT Amsterdam, The Netherlands e-mail: m.d.telman@vu.nl

E. A. Holmes

MRC Cognition and Brain Sciences Unit, Cambridge, UK
Keywords Cognitive bias modification - Interpretational style $\cdot$ Adolescence $\cdot$ Anxiety $\cdot$ Stress reactivity

\section{Introduction}

Anxiety conditions are common [1]; disabling for individuals and families; and pose huge costs for society [2]. Adults with life-long, persistent anxiety disorders often report emergence of these problems in adolescence [3]. Indeed, early-emerging anxious problems predict greater risk for later anxiety disorders [4], as well as more serious mental health outcomes, such as substance misuse [5, 6] and suicide [7]. Treatments for early anxiety problems may prevent the persistence of primary and secondary disorders. Yet many current frontline treatments for adolescent anxiety either yield variable outcomes or raise concerns about long-term viability. Moreover, these are financially costly and difficult to access. Recent work from adults has highlighted the potential of Cognitive Bias Modification of Interpretations (CBM-I) training techniques in anxiety reduction. CBM-I targets biases in interpretational style that may be causally linked to anxiety disorders [8], by shaping the tendency to draw positive (or benign) interpretations of ambiguous social situations, through training.

This work, while generating much excitement, has only recently been extended to children [9-14] and adolescents [1517]. Although at present little is known about how interpretational biases to threatening information develop [18], these biases appear susceptible to experimental modification in children as young as 6 years old [12]. However, we believe it is important to extend the work to adolescents for two reasons. First, many anxiety problems onset in the transition to adolescence, and developmental immaturities in cognitive and neurobiological functioning have raised questions over whether current psychological and pharmacological therapies are 
suitable in these age groups [19, 20]. In contrast, CBM-I, which relies on simple reinforcement-learning mechanisms (pairing a positive outcome/explanation with an ambiguous event), may be similar to the social learning mechanisms by which cognitive biases are first acquired (pairing a negative outcome/explanation with an ambiguous eventoften through verbal report from parents) [21]. Second, the capacity to acquire more adaptive interpretative styles may be stronger in adolescence, as this age range overlaps with critical periods of protracted brain maturation and associated plasticity [22]. Indeed, cognitive styles become increasingly stable, cohesive and trait-like in the transition to adolescence [23], flagging this as a developmentally-opportune period for training-facilitated learning.

Drawing on CBM-I training methods used in adults [24, 25], we and others have tried to experimentally manipulate adolescent interpretational styles using computerized paradigms to assess their effects on changes in various mood-states including anxiety, low mood, negative affect (a combination of the two) and positive affect [15-17]. As with the original adult studies, adolescent participants were given short emotional but age-appropriate ambiguous scenarios to read. These scenarios were disambiguated by completing a word fragment. There was only one correct solution to each word fragment: in the positive CBM-I condition, word completion always resulted in a positive interpretation of the situation while, in a comparison, negative CBM-I condition, this resulted in a negative interpretation. After a series of training trials, participants were administered an interpretation bias test, which measured endorsement of positive and negative interpretations of new ambiguous situations. Participants also completed pre- and post-training measures of the different mood-states to assess training-linked changes.

Paralleling adult findings, we and others have found in three independent samples that adolescents who received positive CBM-I endorsed more positive interpretations and fewer negative interpretations of new ambiguous material than those who received negative CBM-I [15-17]. These data clearly demonstrate that positive interpretational styles can be shaped in adolescents. However, more problematically, was the lack of consistency with which training also induced changes on moodstates. In only one of these studies, was a significant reduction in negative affect found in those who had received positive training [16]. In the second and third study, changes in affect were either only found in negatively-trained individuals (those with low self-efficacy) [15], or not at all [17]. Thus the effect of shaping positive interpretational style on adolescent moodstates linked to anxiety remains to be clarified, an important issue for establishing whether positive interpretations can be used to protect against negative outcomes.

Adult data also reveals conflicting results. While some find training effects on anxious, low mood or a combination of both [26, 27], others do not [28-31]. Still others report mixed results [32-34]. One explanation why effects of CBM-I training on mood-states are inconsistent pertains to the role of cognitive biases as stress-diathesis factors [35]: negative interpretations may contribute to anxiety by negatively distorting emotional responses to stress, while positive interpretations may protect against these outcomes by boosting resilience towards stress [36]. Thus one does not expect these differential changes in mood-states after training unless the bias is deployed during a stressful situation [37]. To test these ideas, studies have measured emotional responses to stressful situations following CBM-I training. A wide range of stressors has been used, from insolvable anagrams [36], emotional videos [30], imagined feared situations [38, 39] and negative mood inductions [40]. Not all of these attempts have been successful [34, 41, 42], calling into question the ecological validity of many of these 'stressors'. While these experimentally manipulated 'lab stressors' are informative, an interesting alternative would be to ask participants about their perception of real-world stressors, a method deployed here.

Although the selection of appropriate outcome measures in which to assess the effects of training is crucial, other methodological factors may also enhance the effects of CBM-I training on interpretational style, and associated changes in mood-states. A growing area of focus is the active deployment of mental imagery during training. Encouraging participants to mentally simulate the interpretation scenarios using mental imagery compared to just focusing on verbal processing of scenarios has been shown to facilitate the effects of training on mood change in laboratory studies in adults [26, 33, 40, 43, 44] though effects in children is less clear [14] and requires further exploration. In adults at least, imagery processing of ambiguous scenarios is associated with the amplification of both positive and negative emotions, evoking emotion by directly influencing sensory signals, and by activating memories and reactivating associated feelings with these memories [for a review, see 45].

Because CBM-I studies have great clinical potentialthrough the use of positive training to treat symptoms- the extent to which training is equally effective across individuals is also a crucial question. Adult studies have addressed these questions by assessing whether differences in anxiety proneness facilitates or increases resistance to the effects of positive CBM-I. However evidence is mixed. A recent meta-analysis of CBM-I studies suggested that clinical symptoms did not moderate the effects of CBM-I efficacy on changes in mood-states [25]. However another meta-analysis, albeit on another form of cognitive bias modification training-that of attention-pointed to a somewhat larger effect size in symptomatic individuals [46]. In children, attempts to modify interpretational biases using computerised games also pointed to larger effects in high trait-anxious participants [10], although this was not 
replicated in a subsequent study [13]. Thus, it is unclear whether baseline levels of symptoms enhance or attenuate the effects of CBM-I in adolescents.

The primary aim of this study was to determine whether CBM-I training influenced emotional responses to recent, real-world stress. We compared effects of training on appraisals of recent stressful events, hypothesising that negatively-trained adolescents would provide more negative appraisals while positively-trained adolescents would report more positive appraisals. Given the growing evidence of the importance of mental imagery in bias modification, we adapted our training procedure in line with these more powerful procedures $[26,33,40]$. We also tentatively assessed the role of trait anxiety in moderating the effects of CBM-I training on subsequent interpretational style, changes in negative affect, and on appraisals of recent stressors.

\section{Methods}

Participants and Procedures

Forty-nine adolescents aged 15-18 years old were recruited from secondary schools around Oxfordshire. All participants were fluent in English. Participants were asked if they had a current or a past diagnosis of a mood or anxiety disorder, with those responding in the affirmative being excluded from the study. Adolescents were randomly assigned to receive either positive CBM-I $(N=25)$ or negative CBM-I $(N=24)$ training. Three participants were excluded from the final analysis because of technical difficulties with the task $(N=1)$ and failure to understand the training instructions $(N=2)$. After removal of these participants, a sample of $N=46(N=23$ in each training condition) remained with no between-group differences on gender, age, race, or trait anxiety (all $p>.33$, Table 1 ).

Study procedures are presented in Fig. 1. These were approved by the Central University Research Ethics Committee of the University of Oxford. Participants were not informed of the purpose of the bias modification paradigm until after the study. Participants aged 16 years or above provided informed written consent. Participants under the age of 16 provided written assent and a parent or legal guardian gave informed consent. Subsequently, assessment of trait anxiety was taken using the Trait Anxiety Inventory for Children (STAI-T-C).

Prior to training, participants were taught to use mental imagery through two 'imagery' exercises. These involved describing the sensations of biting into a lemon and coming home from school while being prompted with questions by the researcher ('How does it taste/smell/sound?', 'What can you see/hear/smell?'). Participants were further instructed to use mental imagery during CBM-I training by
Table 1 Participant characteristics and training performance

\begin{tabular}{lll}
\hline & Positive training & Negative training \\
\hline Demographics & & \\
Sample size & 23 & 23 \\
Mean Age (SD) & $16.48(0.99)$ & $16.55(0.74)$ \\
Gender (\%) & 17 female & 19 female \\
& $(73.9 \%)$ & $(82.6 \%)$ \\
& 6 male $(26.1 \%)$ & 4 male $(17.4 \%)$ \\
Ethnicity (\%) & $65.2 \%$ Caucasian & $82.6 \%$ Caucasian \\
& $34.8 \%$ other & $17.4 \%$ other \\
Mean Trait anxiety (SD) & $36.74(7.35)$ & $38.92(7.65)$ \\
Training performance & & \\
Mean CQ ${ }^{\mathbf{a}} \mathrm{RT}^{\mathbf{b}}$ (SD) & $2610.31 \mathrm{~ms}$ & $2646.30 \mathrm{~ms}$ \\
& $(838.23)$ & $(945.36)$ \\
Mean Word fragment RT & $1720.23 \mathrm{~ms}$ & $1902.28 \mathrm{~ms}$ \\
$\quad(\mathrm{SD})$ & $(828.48)$ & $(895.14)$ \\
$\%$ CQ & & $87 \%$ \\
\hline a correct & $91.1 \%$ & \\
b Comprehension questions &
\end{tabular}

reading and imagining each scenario as if it was happening to themselves.

Assessments of negative and positive affect were taken immediately before (T1) and after training (T2) using visual analogue scales (VASs) that contained items from the Positive and Negative Affect Scale for Children [PANASC; 47]. Following training, participants completed a 10-min filler task, to allow groups differences in negative or positive affect that emerged because of training to dissipate [24]. While we were interested in exploring differences in mood-states after training, we did not want these to confound performance on the interpretation bias measure. In other words, it was important to demonstrate changes in interpretational style there were independent from current mood-state. To ensure that the filler task effectively removed differences in mood-state, a third set of VASs measured negative and positive affect after the filler task (T3). Participants then completed the computerized CBM-I test and a Recent Events Checklist to assess training effects on subsequent interpretational style and appraisals of recent stressful events. A final VAS (T4) was then used to assess negative and positive affect. To examine whether participants had inferred the objectives of the study, participants were asked to write down their thoughts on what the study was about. Computerised training was carried out on a laptop computer using E-Prime 2.0.

\section{CBM-I Training Task}

We used a previously developed version of the CBM-I training task for adolescents [16]. The training task consisted of one practice trial followed by 60 trials, presented over 5 
Fig. 1 Overview of experimental procedure. $C B M-I$ cognitive bias modification interpretation training, STAI-T-C State Trait Anxiety InventoryTrait Subscale for Children, $V A S$ visual analogue scale

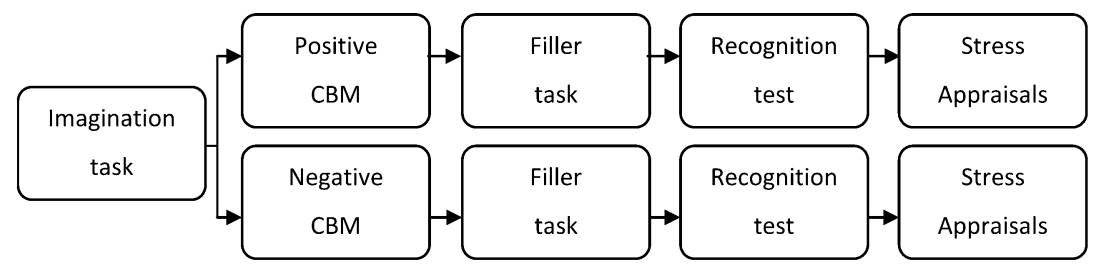

\begin{tabular}{llllll}
\hline $\begin{array}{l}\text { Consent } \\
\text { STAI-T-C }\end{array}$ & VAS1 & VAS2 & VAS3 & VAS4 & $\begin{array}{l}\text { Manipulation } \\
\text { check }\end{array}$ \\
\end{tabular}

blocks. Each trial consisted of a short description of an everyday scenario which remained emotionally ambiguous until the final word, which was presented as a word fragment on the following screen. Participants had to identify the final word as quickly as possible by typing the first missing letter only. Participants could only continue the training task once the word fragment was correctly completed. This word resolved the ambiguity of the scenario, leading to a positive interpretation for most trials in the positive condition, and a negative interpretation for most trials in the negative condition. In each condition, 5 of the scenarios ( 1 in each block of 12 trials) resolved with the opposite valence (negative in the positive condition and vice versa) and 5 of the scenarios (again 1 in each block of 12 trials) resolved neutrally. These items obscured the purpose of the training.

After a participant had completed the first missing letter, he/she was asked to answer a comprehension question for which the correct answer ('Yes' or 'No') was based on the intended emotional interpretation of the training scenario. All comprehension questions were followed by feedback: "Correct" or "Wrong". Therefore both the word fragment completion and comprehension question reinforced the intended emotional valence of the scenario. Participants were asked to respond to the comprehension questions as quickly and accurately as possible.

To illustrate, an example of a training item is: During maths, you are asked by the teacher to write the homework on the board. When you have finished, your teacher looks at it with an expression of agr-m-nt (positive training condition) or disagr-m-nt (negative training condition). In both cases, the correct letter to type in is ' $e$ '. The comprehension question following completion of this word with the letter ' $\mathrm{e}$ ' is: Does your teacher approve of your work? The correct response for those in the positive training is Yes and for those in the negative training, No. The order of scenarios within each block was randomised across participants.

Filler Task

The filler task comprised a picture rating task in which participants rated 60 emotionally neutral pictures on a VAS between 'very unpleasant' $(0 \mathrm{~cm})$ and 'very pleasant' $(9.8 \mathrm{~cm})$.

Assessment of CBM-I Training Effects

on Interpretational Style

The measure of subsequent interpretational style posttraining consisted of two phases [16, 24]. First, ten ambiguous scenarios were presented, each with a title. Participants were given the same instructions as in the training phase. This time, completing word fragments did not disambiguate the emotional valence of the scenarios and the comprehension questions had no emotional content. In the second phase, participants viewed the title of each scenario, followed by four statements relating to that scenario. Participants were informed that these differed in their resemblance to the scenario, but none would be an exact match. They had to rate statements on their similarity to the scenarios viewed in the first phase on a scale from 1 to 4 (1: not similar at all; 2: not so similar; 3 : similar; 4 : very similar). These statements, ordered randomly, included one positive and one negative interpretation of the scenario (targets). The other two statements were also positive and negative but were not interpretations (foils). Bias induction was successful if the negative bias induction group rated the negative targets as more similar to the original ambiguous scenarios, and the positive induction group rated the positive targets as more similar [24]. Similarity of foils assessed the degree to which the training induced a general affective bias towards items of a particular valence.

Assessment of CBM-I Training Effects on Changes in Negative and Positive Affect

Negative and positive affect was assessed at four timepoints during the study (T1-T4) using VAS versions of items drawn from the PANAS-C [PANAS-C; 47]. Eight emotions formed the negative affect VAS and contained items relating to anxious and low mood (nervous, sad, upset, worried, anxious, miserable, scared, gloomy) while 
four items formed a positive affect scale (happy, calm, cheerful, energetic). Participants indicated on a line how much of that emotion they were feeling at that moment between "not [emotion] at all" $(0 \mathrm{~cm})$ and "very [emotion]" $(9.8 \mathrm{~cm})$. VASs can be effective in detecting the influence of an intervention on a dependent variable [48].

\section{Assessment of CBM-I Training Effects on Appraisals of Recent Stressors}

Participants completed an adapted recent life events scale, which also assessed appraisals of events in terms of perceived impact, coping and controllability. Fifteen items with high face validity were taken from the Adolescent Perceived Events Scale [49] and the Child Life Events Checklist [50] to reflect several domains of stress: family relationships, peer relationships, academic achievements, and uncertainty about the future. For every item, participants indicated whether or not a particular event had happened in the past 6 months. If they answered 'YES', participants had to answer the following three questions using a 4-point Likert scale: (a) "How much impact did this event have on your life"; (b) "How able were you to cope with this event" and (c) "How much did you think you could control this event?". The total number of events experienced was summed for each participant. In addition, mean 'impact', 'coping' and 'controllability' scores were calculated for events experienced for each participant.

\section{Trait Anxiety}

All participants completed the Trait Anxiety Inventory for Children [STAI-T-C; 51] prior to computerized training. This is a self-report measure of 20 items, in which participants rate how frequently anxiety symptoms apply to them. Items are summed to create a total anxiety score. This measure has high internal reliability (Cronbach's $\alpha=.91$ ) and correlates well with other measures of child and adolescent anxiety [52]. A median split on this measure $($ median $=38.5)$ yielded low and high anxious groups.

\section{Statistical Analyses}

All statistical analyses were performed in SPSS 17.0 (Chicago, IL, UAS). All paired—and independent—sample $t$ tests were two-tailed. Wherever assumptions of normality were violated, $\log$ transforms were used in the analysis. First, independent sample $t$ tests were carried out to assess group differences in training-related task performance including Reaction Times (RTs) to completion of word fragments and comprehension questions and the percentage of correctly answered comprehension questions. Second, to examine training effects on interpretations of new ambiguous material, we first conducted a $2 \times 2 \times 2 \times 2$ mixed measures analysis of variance (ANOVA) to assess the effects of two within-subjects factor (recognition statement type: target versus foil; recognition statement valence: positive vs. negative) and two between-subjects factors (training group: positive vs. negative and trait anxiety: high vs. low defined by a median split variable) on similarity ratings. As preliminary analysis confirmed a significant main effect of recognition statement type $(p<0.001)$ and a significant 3-way interaction between recognition statement type, recognition statement valence and training group $(p<0.001)$, we conducted separate $2 \times 2 \times 2$ ANOVAs for target and foil ratings. As preliminary analysis showed no main effects of age and gender on similarity ratings, these variables were not included in the final models.

Third, independent sample t tests were used to assess differences in negative and positive affect at $\mathrm{T} 1$ to assess for group differences at baseline; at $\mathrm{T} 3$ to assess for group differences before the interpretation bias measure; and at T4, to assess residual mood differences towards the end of testing. Finally, two $2 \times 2 \times 2$ mixed measures ANOVAs were performed on the data on negative and positive affect with time as a within subjects-factor (T1 vs. T2); training group (positive vs. negative) and trait anxiety (high vs. low) as between-subjects factors. No main effects of age and gender precluded these variables from being entered in final analyses. Appraisals of real-world stressors were assessed using a series of 2-way ANOVAs with training condition and trait anxiety as the between-groups factors. The main dependent variables were total reported events and the three stress appraisals: impact, coping, and controllability.

\section{Results}

\section{Task Performance During CBM-I Training}

No significant differences were found between the training conditions on average RTs to word fragments $[\mathrm{t}(44)=.72$, $p=\mathrm{n}$.s. $]$ or to comprehension questions $[\mathrm{t}(44)=.14$, $p=$ n.s.]. There were also no training differences on comprehension question accuracy $[\mathrm{t}(44)=1.67, p=$ n.s.] (Table 1), suggesting similar task performance across training groups.

\section{Effects of CBM-I on Interpretation Style}

As our first mixed-measures ANOVA analysis showed a significant 3-way interaction between recognition statement type, recognition statement valence and training 
group $[\mathrm{F}(1,42)=15.67, p<.001]$, we conducted separate $2 \times 2 \times 2$ ANOVAs for target and foil ratings. Specifically, we assessed the effects of training-group (positive, negative) on statement-valence (positive, negative) across anxiety-group (high, low). The mixed-measures ANOVA for targets showed only a significant training-group-bystatement-valence interaction $[F(1,42)=30.18, p<.001$, $\eta_{p}^{2}=.42$ ] (Fig. 2). To decompose this we assessed training differences on positive targets and negative targets separately. All between-group and within-group comparisons were significant. The positive training group gave higher similarity ratings to positive targets than the negative training group [ $t(42)=3.83, p<.001$, cohen's $d=1.15]$ but negatively-trained participants gave higher similarity ratings to negative targets than the positively-trained participants $[t(42)=3.52, p<.001$, cohen's $d=1.07]$. The positive CBM-I group also rated positive targets more similar to the ambiguous situations than negative targets $[t(20)=4.72, \quad p<.001$, cohen's $d=1.41]$. For the negative CBM-I group the opposite effect emerged $[t(22)=3.11, p<.01$, cohen's $d=.85]$. None of the differential effects of training were moderated by anxiety group. Nor were there main effects of trait anxiety.

For foils a significant training group-by-statementvalence interaction was also found $[F(1,42)=8.45$, $p<.01, \eta_{p}^{2}=.17$ ] (Fig. 2). Further analysis showed that participants who received positive training reported higher similarity ratings for positive foils than negatively-trained adolescents $[t(42)=3.99, p<.001$, cohen's $d=1.20]$ but this between-group difference did not emerge for negative foils. The positive training group also rated positive foils as more similar to the ambiguous situations than negative foils $[t(20)=4.43, p<.001$, cohen's $d=1.30]$ but within-group effects did not emerge for the negative group. Positive foils were more generally endorsed, reflected in a

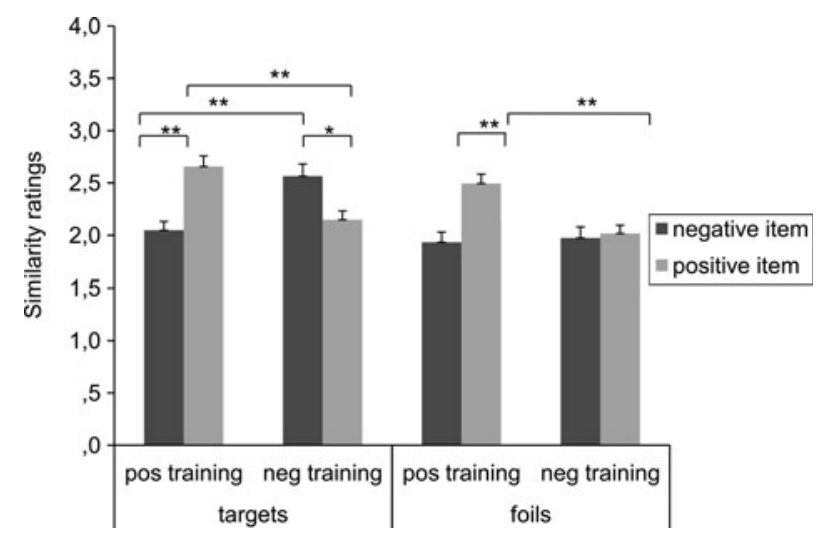

Fig. 2 Effects of training on interpretation style. Similarity ratings for positive and negative targets and foils across training groups in the testing phase. Higher similarity ratings reflect greater resemblance of the item to the ambiguous situation. $* p<.01 ;{ }^{* *} p<.001$ main effect of foils $\left[F(1,42)=11.52, p<.01, \eta_{p}^{2}=.22\right]$. Again, there were no main or interaction effects of trait anxiety group.

Effects of CBM-I on Changes in Negative and Positive Affect

Raw means and standard deviations of the negative and positive affect ratings pre- and post-training across each training condition are shown in Table 2 . As the negative affect ratings at $\mathrm{T} 1-\mathrm{T} 4$ were skewed, $\log$ transformations were used for this outcome at all time-points prior to analysis.

ANOVAs showed a significant training group-by-time interaction $\left[F(1,44)=9.82, p<0.01, \eta_{p}^{2}=.18\right]$ for negative affect. Negative mood increased for the negative training group from $\mathrm{T} 1$ to $\mathrm{T} 2[t(22)=2.51, p<.05$, cohen's $d=.42]$ but this did not change for positivelytrained adolescents. Critically, there were no differences in negative and positive affect between training groups at T1 (baseline) and T3 (prior to interpretation bias test) (Table 2). Unexpectedly, however, a comparison of negative affect at $\mathrm{T} 4$ yielded significant differences $[t(43)=2.18, p<.05$, cohen's $d=.65]$, such that those in the negative training condition reported more negative affect than those in the positive condition.

For positive affect, no significant interaction effect was found $[\mathrm{F}(1,44)=1,99, p=$ n.s.]. Instead, a significant main effect of time emerged $[F(1,44)=8.73, p<0.01$, $\left.\eta_{p}^{2}=.17\right]$, reflecting a decrease in positive affect over time across everyone $(\mathrm{T} 1=5.9, \quad \mathrm{SD}=1.54 ; \quad \mathrm{T} 2=5.28$, $\mathrm{SD}=1.88$ )

Trait anxiety group did not exert main effects on negative and positive affect, nor were there significant effects

Table 2 Mood ratings

\begin{tabular}{|c|c|c|c|c|}
\hline & $\mathrm{T} 1$ & $\mathrm{~T} 2$ & T3 & $\mathrm{T} 4$ \\
\hline \multirow{4}{*}{$\begin{array}{l}\text { Positive } \\
\text { training }\end{array}$} & Positive mood & & & \\
\hline & $5.92(1.77)$ & $5.59(1.95)$ & $5.77(1.67)$ & $5.58(1.81)$ \\
\hline & Negative mood & & & \\
\hline & 1.97 (1.67) & 1.68 (1.47) & 1.67 (1.76) & $1.47(1.57)^{b}$ \\
\hline \multirow{4}{*}{$\begin{array}{l}\text { Negative } \\
\text { training }\end{array}$} & Positive mood & & & \\
\hline & $5.89(1.31)$ & $4.97(1.80)$ & $5.50(2.02)$ & $4.96(2.08)$ \\
\hline & Negative mood & & & \\
\hline & $2.42(1.82)^{\mathrm{a}}$ & $3.18(1.82)^{a}$ & $2.43(1.80)$ & $2.65(2.02)^{b}$ \\
\hline
\end{tabular}

Means and SDs of negative and positive mood before training (T1), after training (T2), before recognition test (T3) and after recognition test (T4). All values are given in $\mathrm{cm}$ on visual analogue scales. Higher values indicate greater intensity of the emotion experienced

Bold values indicate significant differences in mood

${ }^{a}$ Within-group comparison across time, $p<0.05$

b Between-group comparison within a time-point, $p<0.05$ 
on changes in these mood-states pre- to post-training $(p$ 's $>.21)$.

\section{Effects of CBM-I on Appraisals of Recent Stressors}

There were no differences between training groups in the total number of events reported. The number of recent life events varied between 4 and 12, with a mean of 8.33 $(\mathrm{SD}=2.00)$. Analyses showed a main effect of training on impact ratings $\left[F(1,39)=5.39, p<.05, \eta_{p}^{2}=.12\right]$ with negatively-trained individuals rating events as having a greater impact than positively-trained adolescents $[t(41)=$ 2.23, $p<.05$, cohen's $d=.69$; means $=2.69(.49)$ and 2.38(.40) for negative and positive groups respectively]. Training did not influence appraisals of controllability and coping ( $p$ 's $>.10)$.

Trait anxiety influenced ratings of impact $[F(1,39)=$ $\left.6.89, p<.05, \eta_{p}^{2}=.15\right]$; coping $[F(1,39)=9.40, p<.01$, $\left.\eta_{p}^{2}=.19\right]$ and controllability $[F(1,39)=13.03, p<.01$, $\left.\eta_{p}^{2}=.25\right]$. Compared to low-trait anxious participants, those with high-trait anxiety perceived events as having more impact $[$ means $=2.72(.46)$ and 2.37(.42) for high and low groups respectively, $t(41)=2.58, p<.05$, cohen's $d=.79$ ]; were less able to cope with events [means $=2.61(.43)$ and 2.97(.34) for high and low groups respectively, $t(41)=3.06, p<.01$, cohen's $d=.93$ ] and found them less controllable [means $=2.32(.31)$ and 2.76(.46) for high and low groups respectively, $t(41)=$ $3.71, p<.01$, cohen's $d=1.12$ ]. Interactions between training and anxiety group on all three appraisal indices were not significant (all $p$ 's $>.27$ ).

\section{Discussion}

The present study assessed the effectiveness of an adolescent version of a CBM-I training task in producing alterations in interpretation biases, changes in negative affect, and emotional responses to real-life stressors. This task drew on adult CBM-I studies that deployed mental imagery to enhance the effects of negative and positive training [26, $33,40]$. A secondary aim was to explore the role of trait anxiety in moderating the effects of training. This study yielded several new findings. First, we provided clear support for our previous findings $[15,16]$, that negative and positive interpretation biases can be induced in adolescents by current computerized training methods. Second, differential effects of training on negative affect emerged: negative CBM-I training predicted elevations in negative affect across participants, while positively-trained individuals showed no change on this measure. Neither training shifted positive affect. However, negative and positive CBM-I training differentially altered appraisals of recent stressors, with positively-trained adolescents rating these as being less impacting. Crucially, no difference in the number of recent stressors was reported across groups. Training effects on interpretation bias, negative affect, and emotional responses to stress did not vary by trait anxiety.

While some exciting implications can be drawn from these data, they should also be interpreted in light of some limitations. First, we included no baseline measure of interpretation bias. Thus one cannot discount the possibility that the two training groups were not matched on interpretation bias prior to training, confounding subsequent group differences. Moreover, including baseline assessments would allow one to explore whether changes associated with training were due to: (1) positive training elevating positive interpretative styles; (2) positive training reducing access to negative interpretative styles; (3) negative training increasing availability of negative interpretational styles; or (4) negative training reducing access to positive interpretational styles. As the measure of interpretation bias used here involves a recognition test, administering this before and after training would have influenced participants' performance on the second test occasion. A suggestion for future research would be to use a different measure of interpretation bias, or to include a no-training or neutral-training control group.

Second, because of time constraints, we only used brief self-reported instruments to assess negative and positive affect, and emotional responses towards stress. Moreover, measures of stress appraisal were retrospective, administered at one time-point only. Even though the two groups did not differ in the number of recent events encountered, we cannot discount the possibility that life events experienced by the negatively-trained group of adolescents were in fact more severe in nature. Nor can we ignore the possibility that negatively-trained adolescents were more likely to retrieve mood-congruent information when asked to appraise recent life events particularly as these adolescents reported more negative affect immediately before the reappraisal exercise. Nevertheless, as our data provides preliminary support for this hypothesis-that CBM-I training may alter stress responding-albeit using retrospective measures as a cruder proxy, the next step of this research will be to use 'prospective' psychophysiological indicators of affectivity and emotional reactivity. One would ideally assess these stress indices before, during and after real-life stressful events [e.g., 53] and compare the rates of change across training conditions.

Finally, while we have shown that CBM-I training can produce post-training differences in interpretational style, changes in negative affect, and appraisals of the impact of stressors, we cannot make inferences about the long-term duration of these effects or their generalizability to clinical samples. Before clinical implementation of CBM-I are 
considered, future studies need to follow up these group differences, perhaps in the first instance $24 \mathrm{~h}$ later [31]. Also, while we explored the moderating effects of trait anxiety on training effects, these effects are nevertheless studied in a community sample, with 'normally-varying' anxiety levels. Examining these hypotheses in clinical groups of either anxiety is a prerequisite to examining whether CBM-I training is a feasible therapeutic tool.

In spite of these caveats, taken in the context of our previous results, these data are promising in showing that CBM tasks can produce different negative and positive interpretational styles in adolescents through training. These differences were evident on a well-used test of interpretation bias [24] where, positively-trained adolescents endorsed more positive but fewer negative interpretations of new ambiguous material than adolescents in the negative training condition. Within-group differences were also apparent: positive CBM-I training produced more positive interpretations than negative interpretations, with the reverse being true for negative CBM-I training. Induced biases in the positive CBM-I condition were sufficiently powerful that they transferred to a greater endorsement of positive 'foils', statements that were of a general positive valence. Training findings were not explained by group differences in negative or positive mood-states, as no significant differences emerged in ratings between training groups at the time of bias testing (i.e., T3). Nor were there differences in training ability, as indicated by reaction times to completing word fragments and accuracy on comprehension questions.

Our findings also provide tentative support to cognitive theories proposing a causal link between negative interpretational style and symptoms of anxiety. Trained interpretational styles had differential effects on changes in negative affect in the short-term: following negative training, we found the predicted elevations in negative affect. While these group differences were deliberately 'normalised' by the neutral filler task, to avoid confounding performance on the interpretation bias test, the elevation in negative affect had returned at $\mathrm{T} 4$, after the test, reflecting some persistence in their effects. In contrast, reductions on the VAS for positive affect were not significant; nor did positive training reduce negative affect. It may be that among non-clinical samples with a higher baseline positive mood, it is easier to induce a deterioration rather than an elevation in mood.

These inconsistencies in results for negative and positive affect, in addition to mixed results reported in prior data in both adolescents and adults, have led others to examine whether differential effects of training on mood are better captured during provocation by a stressor [30, 38, 39, 54]. Indeed, strikingly, we found that training influenced emotional responses to stress through participants' appraisals of recent stressful events. Participants who were trained to interpret events in a negative manner reported greater perceived impact of experienced real-world stressful events than those receiving positive CBM-I. These effects occurred in the absence of a group difference in the overall number of events reported. However as they did occur in the context of training-related differences in negative affect, speculatively these differences may have served to influence cognitive appraisals of recent stressors, explaining the group differences in impact ratings between training conditions. As trait anxiety also significantly but independently affected impact ratings, this appraisal measure may well probe aspects of vulnerability associated with anxiety. Although we found an influence of training on perceived impact of stressful events, it is important to note that training effects did not generalise to perceived controllability or coping to these reported events. Given that the goals of training were to resolve ambiguous situations either in a positive or negative direction, it may be that appraisals of coping and uncontrollability involved bringing to mind the presence of more specific eventrelated behaviours (e.g., I remember going out more with my friends to cope with my parents' divorce), which reduced the effects of training on these measures. In contrast, appraisals of impact may form a more global appraisal of stressful life events, which resonated more strongly with training goals. However, these interpretations are highly speculative and require further replication with more refined measures of stress appraisal to tease these differences in results apart.

While these data may speak tentatively to the role of negative interpretative biases preceding anxiety, findings that trained positive interpretative styles attenuate stress appraisals are a useful starting point for the development of new clinical initiatives. As anxiety appears to increase in the adolescent years and many adult forms of these disorders start early in life [55], adolescence is an important target for early clinical interventions, particularly with an increasing focus on prevention rather than simply treatment. While psychological treatments are commonly used in young people, there are also issues with access [56]. Moreover, there may be reluctance among some adolescents to engage in face-to-face talking therapy. Developing new tools that utilise new technologies such as interactive, online computer games, as a 'cognitive vaccine' against stress [40] may be an alternative worth pursuing. In this study, high levels of trait anxiety did not affect resistance to these training methods. In considering additional factors that may enhance the effects of training, adult data point to training tasks that incorporate the use of mental imagery. Specifically, these may help to increase the personal salience of the training scenarios. While we did not compare training with and without mental imagery in this study, 
future CBM-I studies of adolescents may wish to investigate benefits (or costs) associated with this additional manipulation.

\section{Summary}

Our findings contribute to a growing body of evidence that suggest the plasticity of cognitive biases in youth. This malleability can be achieved through a computerised procedure involving repeated exposure to the reinforced positive and negative resolution of imagined ambiguous situations. Given the scale of adolescent anxiety and its impact, fresh innovations for therapy are in demand. However future research first needs to address the potential long-term clinical benefits of CBM-I training more rigorously.

Acknowledgments The authors are grateful to all students that participated in the study. The authors want to thank Belinda Platt for assistance with data collection and Claudia Lothmann for her help with preparation of the test materials. M.D. Telman was supported by an Erasmus Student Grant. J.Y.F. Lau is supported by The British Academy. E.A. Holmes is supported by the Wellcome Trust Clinical Fellowship (WT088217), The Medical Research Council, The Lupina Foundation, and the National Institute for Health Research (NIHR) Oxford Biomedical Research Centre based at Oxford University Hospitals Trust Oxford University. The views expressed are those of the author(s) and not necessarily those of the NHS, the NIHR or the Department of Health.

Open Access This article is distributed under the terms of the Creative Commons Attribution License which permits any use, distribution, and reproduction in any medium, provided the original author(s) and the source are credited.

\section{References}

1. Kessler RC et al (2007) Lifetime prevalence and age-of-onset distributions of mental disorders in the World Health Organization's World Mental Health Survey Initiative. World Psychiatry 6(3):168-176

2. Greenberg PE et al (1999) The economic burden of anxiety disorders in the 1990s. J Clin Psychiatry 60(7):427-435

3. Gregory AM et al (2007) Juvenile mental health histories of adults with anxiety disorders. Am J Psychiatry 164(2):301-308

4. Pine DS et al (1998) The risk for early-adulthood anxiety and depressive disorders in adolescents with anxiety and depressive disorders. Arch Gen Psychiatry 55(1):56-64

5. McKenzie M et al (2010) Association of adolescent symptoms of depression and anxiety with daily smoking and nicotine dependence in young adulthood: findings from a 10-year longitudinal study. Addiction 105(9):1652-1659

6. Zimmermann P et al (2003) Primary anxiety disorders and the development of subsequent alcohol use disorders: a 4-year community study of adolescents and young adults. Psychol Med 33(7):1211-1222
7. Nock MK et al (2009) Cross-national analysis of the associations among mental disorders and suicidal behavior: findings from the WHO World Mental Health Surveys. Plos Med 6(8):1-17

8. MacLeod C, Koster EHW, Fox E (2009) Whither cognitive bias modification research? Commentary on the special section articles. J Abnorm Psychol 118(1):89-99

9. Vassilopoulos SP, Banerjee R, Prantzalou C (2009) Experimental modification of interpretation bias in socially anxious children: Changes in interpretation, anticipated interpersonal anxiety, and social anxiety symptoms. Behav Res Ther 47(12):1085-1089

10. Muris $P$ et al (2008) A space odyssey: experimental manipulation of threat perception and anxiety-related interpretation bias in children. Child Psychiatry Hum Dev 39(4):469-480

11. Lester KJ, Field AP, Muris P (2011) Experimental modification of interpretation bias regarding social and animal fear in children. J Anxiety Disord 25(5):697-705

12. Lester KJ, Field AP, Muris P (2011) Experimental modification of interpretation bias about animal fear in young children: effects on cognition, avoidance behavior, anxiety vulnerability, and physiological responding. J Clin Child Adolesc Psychol 40(6): 864-877

13. Muris P et al (2009) Ground control to Major Tom: experimental manipulation of anxiety-related interpretation bias by means of the "space odyssey" paradigm and effects on avoidance tendencies in children. J Anxiety Disord 23(3):333-340

14. Vassilopoulos SP et al (2012) Comparing imagery and verbal training instructions for the experimental modification of interpretation and judgemental bias in children. J Behav Ther Exp Psychiatry 43:594-601

15. Lau JYF et al (2011) The plasticity of adolescent cognitions: data from a novel cognitive bias modification training task. Child Psychiatry Hum Dev 42(6):679-693

16. Lothmann $\mathrm{C}$ et al (2011) Cognitive bias modification training in adolescents: effects on interpretation biases and mood. J Child Psychol Psychiatry 52(1):24-32

17. Salemink E, Wiers RW (2011) Modifying threat-related interpretive bias in adolescents. J Abnorm Child Psychol 39(7): 967-976

18. Field AP, Lester KJ (2010) Is there room for 'development' in developmental models of information processing biases to threat in children and adolescents? Clin Child Fam Psychol Rev 13(4):315-332

19. Muris $P$ (2012) Treatment of childhood anxiety disorders: what is the place for antidepressants? Expert Opin Pharmacother 13(1):43-64

20. Grave J, Blissett J (2004) Is cognitive behavior therapy developmentally appropriate for young children? A critical review of the evidence. Clin Psychol Rev 24(4):399-420

21. Muris P, Field AP (2010) The role of verbal threat information in the development of childhood fear. "Beware the Jabberwock!". Clin Child Fam Psychol Rev 13(2):129-150

22. Gogtay N et al (2004) Dynamic mapping of human cortical development during childhood through early adulthood. Proc Natl Acad Sci USA 101(21):8174-8179

23. Hankin BL (2008) Stability of cognitive vulnerabilities to depression: a short-term prospective multiwave study. J Abnorm Psychol 117(2):324-333

24. Mathews A, Mackintosh B (2000) Induced emotional interpretation bias and anxiety. J Abnorm Psychol 109(4):602-615

25. Hallion LS, Ruscio AM (2011) A meta-analysis of the effect of cognitive bias modification on anxiety and depression. Psychol Bull 137(6):940-958

26. Holmes EA, Mathews A (2005) Mental imagery and emotion: a special relationship? Emotion 5(4):489-497 
27. Salemink E, van den Hout M, Kindt M (2007) Trained interpretive bias: validity and effects on anxiety. J Behav Ther Exp Psychiatry 38(2):212-224

28. Mackintosh B et al (2006) Induced biases in emotional interpretation influence stress vulnerability and endure despite changes in context. Behav Ther 37(3):209-222

29. Teachman BA, Addison LM (2008) Training non-threatening interpretations in spider fear. Cognit Ther Res 32(3):448-459

30. Wilson EJ et al (2006) The causal role of interpretive bias in anxiety reactivity. J Abnorm Psychol 115(1):103-111

31. Yiend J, Mackintosh B, Mathews A (2005) Enduring consequences of experimentally induced biases in interpretation. Behav Res Ther 43(6):779-797

32. Hirsch CR, Hayes S, Mathews A (2009) Looking on the bright side: accessing benign meanings reduces worry. J Abnorm Psychol 118(1):44-54

33. Holmes EA et al (2006) Positive interpretation training: effects of mental imagery versus verbal training on positive mood. Behav Ther 37(3):237-247

34. Salemink E, van den Hout M, Kindt M (2007) Trained interpretive bias and anxiety. Behav Res Ther 45(2):329-340

35. Abela JRZ, D'Alessandro DU (2002) Beck's cognitive theory of depression: a test of the diathesis-stress and causal mediation components. Br J Clin Psychol 41:111-128

36. Mathews A, MacLeod C (2002) Induced processing biases have causal effects on anxiety. Cogn Emot 16(3):331-354

37. Hertel PT et al (2003) Transfer of training emotionally biased interpretations. Appl Cogn Psychol 17(7):775-784

38. Hirsch CR, Mathews A, Clark DM (2007) Inducing an interpretation bias changes self-imagery: a preliminary investigation. Behav Res Ther 45(9):2173-2181

39. Murphy R et al (2007) Facilitating a benign interpretation bias in a high socially anxious population. Behav Res Ther 45(7):1517-1529

40. Holmes EA, Lang TJ, Shah DM (2009) Developing interpretation bias modification as a "cognitive vaccine" for depressed mood: imagining positive events makes you feel better than thinking about them verbally. J Abnorm Psychol 118(1):76-88

41. Standage H, Ashwin C, Fox E (2009) Comparing visual and auditory presentation for the modification of interpretation bias. J Behav Ther Exp Psychiatry 40(4):558-570

42. Salemink E, van den Hout M, Kindt M (2009) Effects of positive interpretive bias modification in highly anxious individuals. J Anxiety Disord 23(5):676-683
43. Holmes EA, Coughtrey AE, Connor A (2008) Looking at or through rose-tinted glasses? Imagery perspective and positive mood. Emotion 8(6):875-879

44. Pictet A et al (2011) Fishing for happiness: the effects of generating positive imagery on mood and behaviour. Behav Res Ther 49(12):885-891

45. Holmes EA, Mathews A (2010) Mental imagery in emotion and emotional disorders. Clin Psychol Rev 30(3):349-362

46. Hakamata Y et al (2010) Attention bias modification treatment: a meta-analysis toward the establishment of novel treatment for anxiety. Biol Psychiatry 68(11):982-990

47. Laurent $\mathbf{J}$ et al (1999) A measure of positive and negative affect for children: scale development and preliminary validation. Psychol Assess 11:326-338

48. Grant $\mathrm{S}$ et al (1999) A comparison of the reproducibility and the sensitivity to change of visual analogue scales, borg scales, and likert scales in normal subjects during submaximal exercise. Chest 116:1208-1217

49. Compas BE et al (1987) Assessment of major and daily stressful events during adolescence: the adolescent perceived events scale. J Consult Clin Psychol 55:534-541

50. Johnson JH, McCutcheon S (1980) Assessing life stresses in older children and adolescents: preliminary findings with the life events checklist. In: Sarason IG, Spielberger CD (eds) Stress and anxiety. Hemisphere, Washington, DC, pp 111-125

51. Spielberger CD (1973) State-trait anxiety inventory for children. Consulting Psychologists Press, Palo Alto, CA

52. Muris $P$ et al (2002) Three traditional and three new childhood anxiety questionnaires: their reliability and validity in a normal adolescent sample. Behav Res Ther 40:753-772

53. Dandeneau S et al (2007) Cutting stress off at the pass: reducing vigilance and responsiveness to social threat by manipulating attention. J Pers Soc Psychol 93:651-666

54. Steinman SA, Teachman BA (2010) Modifying interpretations among individuals high in anxiety sensitivity. J Anxiety Disord 24(1):71-78

55. Lewinsohn PM et al (1993) Adolescent psychopathology: I. Prevalence and incidence of depression and other DSM-III-R disorders in high school students. J Abnorm Psychol 102: 133-144

56. Essau CA, Conradt J, Petermann F (2000) Frequency, comorbidity, and psychosocial impairment of anxiety disorders in German adolescents. J Anxiety Disord 14(3):263-279 